This is the final peer-reviewed accepted manuscript of:

D. Cornigli, S. Reggiani, A. Gnudi, E. Gnani, G. Baccarani, D. Fabiani, D. Varghese, E. Tuncer, S. Krishnan, L. Nguyen,

Electrical characterization of epoxy-based molding compounds for next generation HV ICs in presence of moisture

Microelectronics Reliability, Volumes 88-90, 2018, Pages 752-755, ISSN 0026-2714

The final published version is available online at:

https://doi.org/10.1016/j.microrel.2018.07.030

Rights / License:

The terms and conditions for the reuse of this version of the manuscript are specified in the publishing policy. For all terms of use and more information see the publisher's website.

This item was downloaded from IRIS Università di Bologna (https://cris.unibo.it/)

When citing, please refer to the published version. 


\title{
Electrical characterization of epoxy-based molding compounds for next generation HV ICs in presence of moisture
}

\author{
D. Cornigli ${ }^{\mathrm{a}}$, S. Reggiani ${ }^{\mathrm{a}, \mathrm{b}}$, A. Gnudi ${ }^{\mathrm{a}, \mathrm{b}}$, E. Gnani ${ }^{\mathrm{a}, \mathrm{b}}$, G. Baccarani $\mathrm{i}^{\mathrm{a}, \mathrm{b}}$,

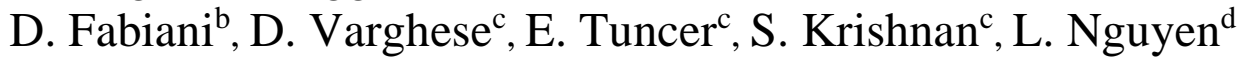 \\ a ARCES, University of Bologna, Bologna, Italy \\ ${ }^{\mathrm{b}}$ DEI, University of Bologna, Bologna, Italy \\ c Texas Instruments, Inc., Dallas, TX 75243, USA \\ ${ }^{\mathrm{d}}$ Texas Instruments Inc., Santa Clara, CA 95051, USA
}

\begin{abstract}
The effects of moisture on the electrical properties of epoxy molding compounds containing high quantities of silica filler microparticles have been investigated by means of dielectric spectroscopy, steady-state conductivity and pulsed electro-acoustic space charge measurements. It has been shown that the presence of water at the filler/epoxy interfacial areas affects the low-frequency dielectric response of the materials and significantly increases their electrical conductivity, especially at higher temperatures. In addition, in presence of moisture, space charge measurements have shown the accumulation of heterocharges which significantly increase the electric field at the electrodes. These aspects are of primary importance, as the electrical properties of the encapsulation materials strongly influence the space charge distribution in high voltage devices.
\end{abstract}

\section{Introduction}

The high demands on weight, cost and size in new power electronic applications is leading to the need of plastic encapsulation materials with improved physical properties. Recently, epoxy-based polymers with a large fraction of insulating fillers have been proposed to decrease moisture permeability and suppress thermal expansion in order to avoid corrosion and exfoliation during high-temperature cycles [1]. In addition, the electrical conductivity is known to be source of charge spreading [2,3]. More specifically, high-voltage lateral devices, like, e.g., double-diffused DMOS (LDMOS) transistors, are based on the Reduced Surface Field (RESURF) principle [4] to significantly improve the breakdown voltage. But the performance of this class of devices is sensitive to the optimum charge balance in the drift region, which is in general at the silicon surface, and thus it is strongly impacted by mobile charges accumulated in the overlying isolation and mold-compound passivation on top [5]. For this reason, additional metal and/or poly-silicon field-plates and floating-rings are required to stabilize the surface electric field [6,7], even if they might limit the maximum intrinsic performance of the device. The understanding of mobile charge transport at the molding compound surface is thus of primary importance to improve the power device performance and reliability at the design stage. Moreover, lifetime tests are usually carried out with humidity as high as $85 \% \mathrm{RH}$. The latter condition would lead eventually to instability and failure of the device just due to the increased electrical transport of the packaging material.

In [8], molding compounds with different composition were investigated to assess the role of insulating fillers on the electrical conductivity, and charge spreading in the encapsulation was characterized on a dedicated IC test chip.

Due to the nature of polymers, water absorption is expected to occur in humid environments, inducing significant changes in the dielectric properties. Recently, polymers with nano-size fillers under moisture conditions has been investigated $[9,10]$. As nanoparticles have significantly different interface features, a specific characterization of micro-filled polymers is still needed.

In this work, the dielectric spectroscopy, DC conductivity and space-charge distribution of polymers with high filler contents as in commercial products have been analyzed under dry and humid conditions. 
The reported data give useful information for assessing the reliability expectations on such composite systems.

\section{Materials and sample characteristics}

The encapsulation materials used in this study are epoxy-based conventional mold compounds (EMCs) employed in semiconductor packaging. The samples are $50 \mathrm{~mm} \times 50 \mathrm{~mm}$ squares with a thickness $h=0.75$ $\mathrm{mm}$. The EMCs are labelled MC0, MC1 and MC2, and contain different fractions of silica micro-fillers. The filler content was measured by thermogravimetric analysis to determine the weight percentage. MC2 has the lowest filler content, while MC1 and MCO contain $20 \%$ and $25 \%$ more filler, respectively.

\section{Humidity absorption}

The moisture absorption of the EMCs has been investigated by exposing the samples to humid conditions in a climatic chamber (Genviro-060-C) with precise control of temperature and relative humidity. As a preliminary treatment, the samples have been dried at $125{ }^{\circ} \mathrm{C}$ for 24 hours and weighted with an analytical balance (Sartorius CP $124 \mathrm{~S}$ ) with an accuracy of $0.0001 \mathrm{~g}$ to determine their dry weight $M_{0}$. Subsequently, the samples have been placed under 85 ${ }^{\circ} \mathrm{C} / 85 \% \mathrm{RH}$ and periodically weighted until equilibrium was reached following [11]. The relative water mass $M_{\%}$ is calculated as:

$$
M_{\%}=100 \times\left(M_{t}-M_{0}\right) / M_{0}
$$

where $M_{t}$ is the weight of a wet specimen at time t. The absorption plot in Fig.1 shows curves similar to literature data $[12,13]$. By assuming that the weight gain of the EMCs initially follows the single-phase

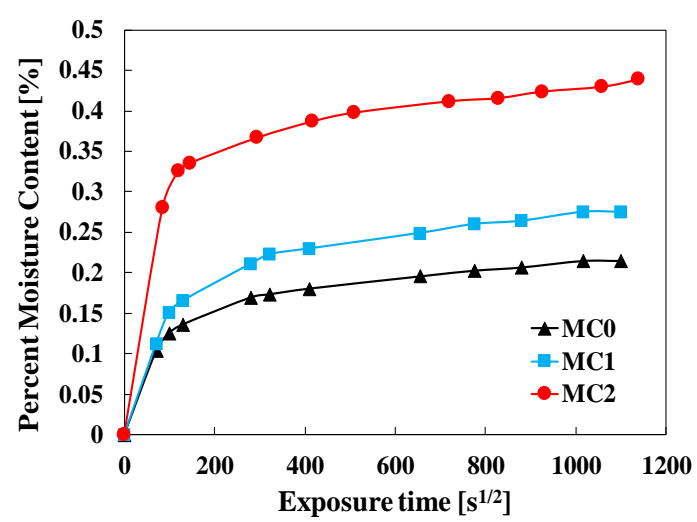

Fig. 1. Water uptake of the EMCs as functions of the square root of time.
Table 1

Diffusivity and moisture equilibrium from Eq. 1.

\begin{tabular}{llll}
\hline & MC0 & MC1 & MC2 \\
\hline $\boldsymbol{D}\left[\mathrm{mm}^{2} / \mathbf{s}\right]$ & $5.1 \times 10^{-6}$ & $4.0 \times 10^{-6}$ & $5.4 \times 10^{-6}$ \\
$\boldsymbol{M}_{\infty}[\%]$ & 0.215 & 0.275 & 0.440 \\
\hline
\end{tabular}

Fickian diffusion model, the diffusivity $D$ has been calculated from the initial gradient of water uptake versus $t^{1 / 2}[11]$ :

$$
D=\pi\left(h / 4 M_{\infty}\right)^{2}\left(M_{2}-M_{1}\right)^{2} /\left(\sqrt{ } t_{2}-\sqrt{ } t_{1}\right)^{2}
$$

where $M_{\infty}$ is the moisture content at equilibrium, $h$ is the thickness of the samples and the last term corresponds to the slope of the moisture plot in the initial linear portion of the curve. The diffusivity and moisture equilibrium content extracted from the absorption plots are reported in Table 1 . It can be noted that the diffusivity ranges between $4.0 \times 10^{-6} \mathrm{~mm}^{2} / \mathrm{s}$ and $5.6 \times 10^{-6} \mathrm{~mm}^{2} / \mathrm{s}$ and seems to be independent from the filler fraction. In contrast, as the quantity of water absorbed by a polymeric material is related to the availability of free volume within the epoxy networks $[14,15]$, significant variations have been measured in $M_{\infty}$ for the different EMCs. In Fig. 2, $M_{\infty}$ is plotted as a function of the filler fraction. The linear correlation between the two quantities is in agreement with similar measurements reported in literature $[12,13]$, confirming that a large fraction of insulating fillers limits the moisture permeability within the epoxy matrix. An opposite trend is obtained for nanocomposites $[9,10]$. . In order to optimize the filler content, additional experiments, such as long-term electro-thermal reliability tests, would be necessary.

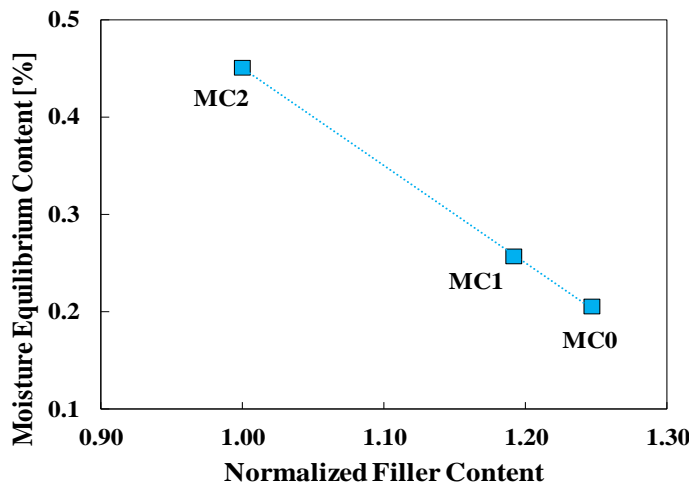

Fig. 2. Equilibrium moisture content at $85{ }^{\circ} \mathrm{C} / 85 \%$ $\mathrm{RH}$ as a function of the normalized filler content of silica with respect to the lowest one. 

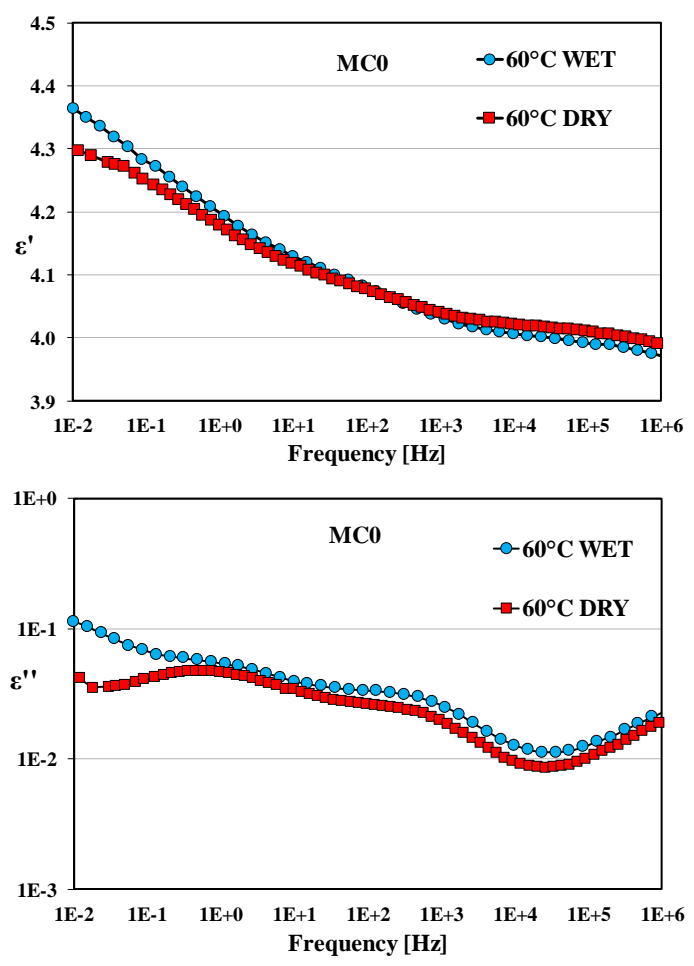

Fig. 3. (top) Permittivity and (bottom) loss factor of $\mathrm{MC} 0$ at $60^{\circ} \mathrm{C}$ in (blue) wet and (red) dry conditions.

However, as the minimum water uptake is shown by $\mathrm{MC0}$, which would assure the best reliability response against corrosion at the IC bond pads [16], the following investigations on charge transport effects have been carried out on this kind of material

\section{Dielectric spectroscopy}

Dielectric analysis (DEA) was carried out on a sample of MC0 both in dry and wet $(0.21 \%)$ conditions. Circular gold electrodes with $40 \mathrm{~mm}$ diameter were sputtered on both sides of the sample and the analysis was carried out by using the Novocontrol Concept 80 alpha analyser. A small AC signal is used $\left(\mathrm{V}_{\mathrm{RMS}}=1 \mathrm{~V}\right)$. The permittivity $\varepsilon$ ' and loss factor $\varepsilon$ " have been measured for frequencies $f$ from $10^{-2}$ to $10^{6} \mathrm{~Hz}$ at $60^{\circ} \mathrm{C}$. In EMCs, the dielectric spectra generally show the effect of epoxy/filler interfacial polarization ( $\varepsilon$ ' at low $f)$, the material conductivity $(\varepsilon$ ', at low $f)$, and the dipolar relaxation times ( $\varepsilon$ " peaks) $[2,17]$. Moisture influences the dielectric properties of EMCs in different ways $[9,18]$. Water molecules in dispersed states can cause a dipolar response, while water clusters or droplets may cause interfacial polarization. As shown in Fig. 3, the wet sample
Table 2

Conductivity measurements of $\mathrm{MC} 0$.

\begin{tabular}{|c|c|c|}
\hline Water content & t $\sigma$ at $25^{\circ} \mathrm{C}[\mathrm{S} / \mathrm{cm}]$ & $\sigma$ at $60{ }^{\circ} \mathrm{C}[\mathrm{S} / \mathrm{cm}]$ \\
\hline $0 \%$ & $7.0 \times 10^{-18}$ & $1.4 \times 10^{-17}$ \\
\hline$\approx 0.1 \%$ & $1.9 \times 10^{-17}$ & $3.5 \times 10^{-17}$ \\
\hline$\approx 0.2 \%$ & $3.2 \times 10^{-17}$ & $2.5 \times 10^{-16}$ \\
\hline
\end{tabular}

exhibits an increase of both $\varepsilon$ ' and $\varepsilon$ ', at low $f$, while no significant differences are observed for $f>1 \mathrm{~Hz}$. This suggests that the presence of water mostly increases the epoxy/filler interfacial polarization and the conductivity without changing the dipolar response.

\section{DC current measurements}

In order to study the relationship between the conductivity and the moisture content, the steady-state current densities have been measured. To this purpose, a current has been induced across the samples by applying a DC step voltage and it has been monitored with a Keithley-6514 electrometer for 1000 seconds into a thermostatic oven. The measurement time is long enough to separate polarization effects and carrier conduction, but short enough to avoid any significant moisture desorption during the measurement. To this purpose, the analysis has been limited to a maximum temperature of $60{ }^{\circ} \mathrm{C}$, which has been checked to give rise to a desorption lower than $15 \%$ of the initial water content, while a larger reduction is expected to affect the validity of the analysis. The conductivity $\sigma$ has been extracted from the steady-state current as:

$$
\sigma=I h /(A V)
$$

where $I$ is the current, $V$ is the applied voltage across the sample, $h$ is the thickness of the sample, $A$ is the area of the electrodes. All the measurements have been obtained by applying a step voltage of $3 \mathrm{kV}$, corresponding to an electric field of about $4 \mathrm{kV} / \mathrm{mm}$ and the experimental setup allows to measure a minimum conductivity about $10^{-19} \mathrm{~S} / \mathrm{cm}$. The analysis has been carried out on a sample of $\mathrm{MC} 0$ with different moisture contents at the temperatures of $25^{\circ} \mathrm{C}$ and 60 ${ }^{\circ} \mathrm{C}$ and the results are reported in Table 2 . It can be noted that the conductivity increases with the moisture content both at $25{ }^{\circ} \mathrm{C}$ and $60{ }^{\circ} \mathrm{C}$. This effect is particularly enhanced at $60^{\circ} \mathrm{C}$, where the conductivity near moisture equilibrium content is about 18 times its dry counterpart. These considerations seem to support 


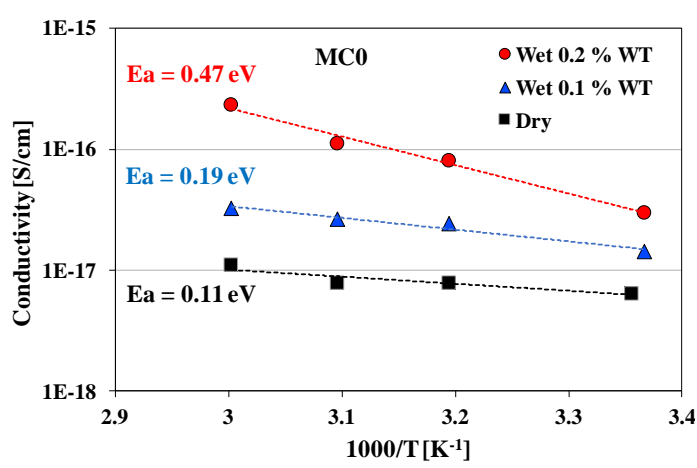

Fig. 4. Reciprocal temperature dependence of the MC0 conductivity at different humidity contents. The corresponding activation energy, $E_{a}$, has been extracted with eq. (4) and significantly increases when the material is in saturation conditions.

the comments on the dielectric responses in Section 4 and are in agreement with the results reported in the literature $[9,10,19]$, where the low-frequency permittivity increases and the measured higher conductivity in wet nanocomposites has been attributed to the presence of water at the filler/epoxy interfacial areas. Within this framework, it is clear that the filler content and its size would affect the way in which water molecules are distributed inside the material. This, in turn, influences in a non-trivial way the conduction mechanisms, and hence the electrical properties of the material. A detailed analysis of these aspects would require a combination of numerical methods and analytical models $[8,20]$

In Fig. 4, the conductivities for different humidity conditions are reported as functions of the reciprocal temperature. It is possible to extract the activation energies corresponding to different humidity contents

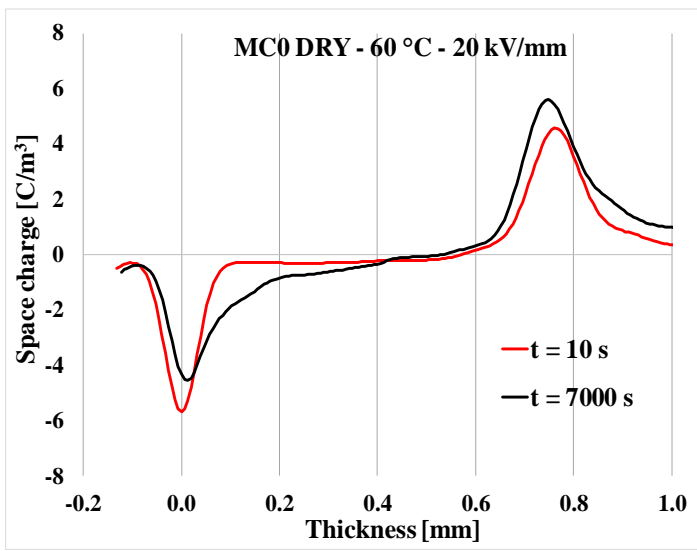

Fig. 5 Space-charge profiles at different times of dry MC0 at $60{ }^{\circ} \mathrm{C}$, with a poling field of $20 \mathrm{kV} / \mathrm{mm}$.

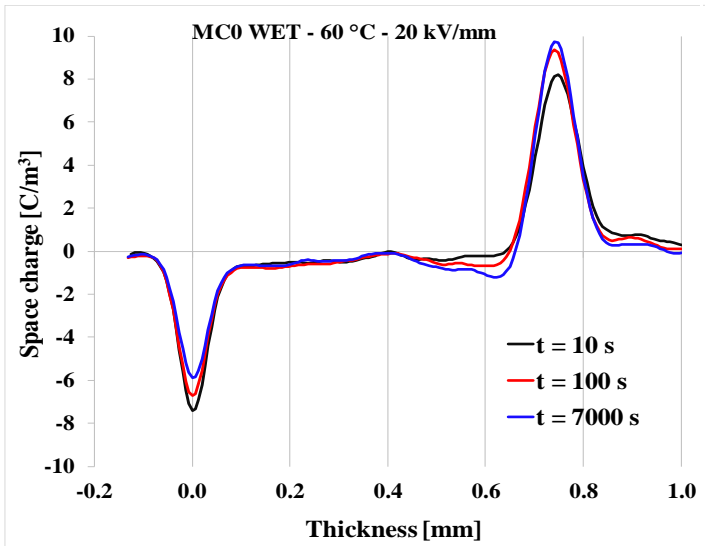

Fig. 6. Space charge profiles at different times of wet $\mathrm{MC} 0$ at $60{ }^{\circ} \mathrm{C}$, with a poling field of $20 \mathrm{kV} / \mathrm{mm}$.

by using the formula:

$$
\sigma=\sigma_{0} \exp \left(-E_{a} / k T\right)
$$

where $\sigma_{0}$ is the pre-exponential term, $E_{a}$ is the activation energy in $\mathrm{eV}, k$ is the Boltzmann constant and $T$ is the temperature in Kelvin. It should be noted that the activation energy of $\sigma$ is strongly influenced by the water content. In particular, it increases non linearly with the water content, passing from $0.19 \mathrm{eV}$ to $0.47 \mathrm{eV}$ as the relative water mass increases from $0.1 \%$ to $0.2 \%$. This behavior is in contrast with what observed in literature for similar materials carried out at temperatures higher than the glass-transition one $[18,21]$. In the present work the analysis is carried out at temperatures significantly lower than the glass transition, which might cause a relevant difference in the involved physical effects and in their thermal dependencies.

\section{Pulsed electro-acoustic analysis}

The Pulsed Electro-Acoustic (PEA) analysis method is used to measure space charge distributions in solid insulating materials, and is based on the acoustic propagation through the material [22]. The samples are $50 \mathrm{~mm} \times 50 \mathrm{~mm}$ squares with a thickness $\mathrm{h}=0.75 \mathrm{~mm}$, without metallization. The space charge distribution of a sample of $\mathrm{MC} 0$ has been measured at $60^{\circ} \mathrm{C}$ for 7000 $\mathrm{s}$ in presence of a poling field of $20 \mathrm{kV} / \mathrm{mm}$. Again, the wet sample refers to a moisture content of about 0.21 $\%$ wt, i.e., close to moisture equilibrium content. Space charge profiles at $10 \mathrm{~s}$ and $7000 \mathrm{~s}$ of polarization on dry and wet specimens are shown in Figures 5 and 6, respectively. It can be noticed that a significant amount 
of negative homocharge is accumulated close to the cathode of the dry specimen, due to charge injection from the electrode, as shown in Fig. 5. In the case of the wet specimen, the negative space charge turns into heterocharge accumulation close to the anode, as shown in Fig. 6. This behaviour could be associated with the increased conductivity of the wet specimen, which favours the transport of injected charge from the cathode to the anode and/or enhances the conductivity of ionic species [23]. This heterocharge build-up can enhance the local field at the electrode/insulation interface (see the increase of the anode peak at $7000 \mathrm{~s}$ ), thus constituting a possible critical issue for the electrical insulation [24].

\section{Conclusions}

Moisture in highly-filled EMCs causes a significant increase in the conductivity, of about a factor 18 at $60{ }^{\circ} \mathrm{C}$, with $0.2 \%$ water uptake, reached at $85 \mathrm{C} / 85 \%$ in 178 hours. However, a water uptake as high as $0.19 \%$, which is expected to increase the conductivity of a similar amount, is measured in less than 48 hours. Moreover, charge injection is improved in the presence of high fields, leading to charge spreading. As already observed in previous investigations, a modest increase of EMC conductivity leads to a significant increase of the surface potential on top of active regions of semiconductor devices. Thus, it is recommended that the electrical properties of EMCs under moisture condition should be evaluated to mitigate any unexpected degradation in device performance.

\section{Acknowledgments}

This work was supported by Texas Instruments Inc., Dallas, TX.

\section{References}

[1] A. Garcia et al., "Reliability of High-Voltage Molding Compounds: Particle Size, Curing Time, Sample Thickness, and Voltage Impact on Polarization," in IEEE Transactions on Industrial Electronics, vol. 63, no. 11, pp. 7104-7111, Nov. 2016.

[2] I. Imperiale et al., "TCAD modeling of charge transport in HV-IC encapsulation materials," 2014 IEEE 26th International Symposium on Power Semiconductor Devices \& IC's (ISPSD), Waikoloa, HI, 2014, pp. 450453.

[3] G. Arienti et al., "Optimization of HV LDMOS devices accounting for packaging interaction," 2015 IEEE 27th
International Symposium on Power Semiconductor Devices \& IC's (ISPSD), Hong Kong, 2015, pp. 305-308.

[4] A. W. Ludikhuize, "A Review of RESURF Technology," in Proc. Int. Symp. Power Semicond. Devices ICs, 2000, pp. 11-18.

[5] A.S. Chen, A. Shafi, R. W. Busse, R. Orr, R. H. Y. Lo, "Molding compounds for high breakdown voltage applications on power IC semiconductors", Proc. Electronic Components and Technology Conference (ECTC), p. 91, 1997.

[6] Z. Hossain, "Determination of Manufacturing Resurf Process Window for a Robust 700V Double Resurf LDMOS Transistor", Proc. ISPSD 2008, p. 133.

[7] M. Venturato, G. Cantone, F. Ronchi, F. Toia, "A novel $0.35 \mu \mathrm{m} 800 \mathrm{~V}$ BCD technology platform for Offline Applications", Proc. ISPSD 2012, p. 397.

[8] I. Imperiale et al., "Role of the Insulating Fillers in the Encapsulation Material on the Lateral Charge Spreading in HV-ICs," in IEEE Transactions on Electron Devices, vol. 64, no. 3, pp. 1209-1216, March 2017.

[9] L. Hui, J. K. Nelson and L. S. Schadler, "The influence of moisture on the electrical performance of XLPE/silica nanocomposites," 2010 10th IEEE International Conference on Solid Dielectrics, Potsdam, 2010, pp. 1-4.

[10] D. Qiang, Yan Wang, G. Chen and T. Andritsch, "Influence of water absorption on space charge behavior of epoxy nanocomposites," 2016 IEEE Conference on Electrical Insulation and Dielectric Phenomena (CEIDP), Toronto, ON, 2016, pp. 603-606.

[11] ASTM D5229 / D5229M-14, " Standard Test Method for Moisture Absorption Properties and Equilibrium Conditioning of Polymer Matrix Composite Materials" ASTM International, PA, 2004.

[12] S. Zhao, "Moisture absorption of molding compound and organic substrate," 2011 International Symposium on Advanced Packaging Materials (APM), Xiamen, 2011, pp. 387-393.

[13] X. Ma, K. M. B. Jansen, G. Q. Zhang and L. J. Ernst, "Filler Contents Effects on the Moisture Absorption and Viscoelasticity of Thermosetting IC Packaging Polymers," 2006 7th International Conference on Electronic Packaging Technology, Shanghai, 2006, pp. $1-5$.

[14] D.P.N. Vlasveld, J. Groenewold, H.E.N. Bersee, S.J. Picken, "Moisture absorption in polyamide-6 silicate nanocomposites and its influence on the mechanical properties", Polymer, Volume 46, Issue 26, 2005, Pages 12567-12576

[15] L. Li, Y. Yu, H. Su, G. Zhan, S. Li, and P. Wu, "The Diffusion Mechanism of Water Transport in AmineCured Epoxy Networks," Appl. Spectrosc. 64, 458-465 (2010)

[16] P. Lall, Y. Luo and L. Nguyen, "Package-level multiphysics simulation of $\mathrm{Cu}-\mathrm{Al} \mathrm{WB}$ corrosion under high temperature/humidity environmental conditions," 2017 16th IEEE Intersociety Conference on Thermal and Thermomechanical Phenomena in Electronic Systems 
(ITherm), Orlando, FL, 2017, pp. 1176-1184.

[17J. Castellon et al., "Electrical properties analysis of micro and nano composite epoxy resin materials," in IEEE Transactions on Dielectrics and Electrical Insulation, vol. 18, no. 3, pp. 651-658, June 2011.

[18] E. Tuncer, " Change in dielectric relaxation with the presence of water in highly filled composites" Journal of Advanced Dielectrics, Volume 7, n.5, 2017, pp. 1750033-1-11.

[19] E. Tuncer, S. M. Gubanski, J. Lambrecht and R. Barsch, "Thermally stimulated depolarization currents of silicone rubbers immersed in water," Conduction and Breakdown in Solid Dielectrics, 1998. ICSD '98. Proceedings of the 1998 IEEE 6th International Conference on, Vasteras, 1998, pp. 193-197.

[20] W. Ahn et al., "Optimum filler geometry for suppression of moisture diffusion in molding compounds," 2016 IEEE International Reliability Physics Symposium (IRPS), Pasadena, CA, 2016, pp. PA-1-1-PA-1-4.

[21] C. Zou, J. C. Fothergill and S. W. Rowe, "The effect of water absorption on the dielectric properties of epoxy nanocomposites," in IEEE Transactions on Dielectrics and Electrical Insulation, vol. 15, no. 1, pp. 106-117, February 2008.

[22] T. Takada, "Acoustic and optical methods for measuring electric charge distributions in dielectrics," 1999 Annual Report Conference on Electrical Insulation and Dielectric Phenomena (Cat. No.99CH36319), Austin, TX, 1999, pp. 1-14 vol.1.

[23] D. Fabiani, G. C. Montanari and L. Testa, "Effect of aspect ratio and water contamination on the electric properties of nanostructured insulating materials," in IEEE Transactions on Dielectrics and Electrical Insulation, vol. 17, no. 1, pp. 221-230, February 2010.

[24] P. Morshuis, A. Cavallini, D. Fabiani, G. C. Montanari and C. Azcarraga, "Stress conditions in HVDC equipment and routes to in service failure," in IEEE Transactions on Dielectrics and Electrical Insulation, vol. 22, no. 1, pp. 81-91, Feb. 2015. 